Moran, T., Bar Anan, Y., (in press). The Effect of Co-occurrence and Relational Information on Speeded Evaluation. Cognition and Emotion.

\title{
The Effect of Co-occurrence and Relational Information on Speeded Evaluation
}

\author{
Tal Moran
}

Ben-Gurion University of the Negev, Beer-Sheva, Israel

Ghent University, Belgium

Yoav Bar-Anan

Ben-Gurion University of the Negev, Beer-Sheva, Israel

Tel-Aviv University, Israel

Author's note: Correspondence should be addressed to: Tal Moran, Department of Experimental Clinical and Health Psychology, Ghent University, Belgium. Email: Tal.MoranYorovich@UGent.be. This work was supported by grants from the Israeli Science Foundation [779/16] and the United States-Israel Binational Science Foundation [2013214] to Y. B.A. 


\begin{abstract}
After co-occurrence of a neutral conditioned stimulus (CS) with an affective unconditioned stimulus (US), the evaluation of the CS acquires the US valence. This effect disappears when information about the CS-US relation indicates that they are opposite in valence. In that case, people often show a contrastive effect, evaluating the CS with valence opposite of the US. We investigated whether the assimilative effect of co-occurrence persists and is only obscured by a stronger counteracting contrast effect of the inference from the CS-US opposition relation. Participants evaluated CSs that had opposite relations with the USs under time pressure, a condition that restricts inference processes more than it restricts the associative processes that might underlie the assimilative effect of co-occurrence. Evidence supporting the persistence of the assimilative effect emerged only in Experiment 2 (N =79): Under time pressure, people evaluated creatures that ended positive sounds more favorably than creatures that ended negative sounds. However, no difference between the creatures' evaluations occurred under time pressure in Experiments 1 and $3(\mathrm{Ns}=78,460)$. These results are inconclusive because they might reflect equal contrastive and assimilative effects or no effects at all. We discuss further research directions to test our question.
\end{abstract}

Key words: Evaluative conditioning, Co-occurrence, Relational information, Speeded evaluation 


\section{The Effect of Co-occurrence and Relational Information on Speeded Evaluation}

People often encounter novel or neutral objects that co-occur with affective stimuli. For example, a new brand may co-occur with a popular celebrity on TV. Previous studies found that when a neutral conditioned stimulus (CS) repeatedly occurs with a positive or a negative unconditioned stimulus (US), the common result is an assimilative effect: the evaluation of the formerly neutral stimulus shifts toward the valence of the affective stimulus, an effect known as Evaluative Conditioning (EC; De Houwer, Thomas, \& Baeyens, 2001; Hofmann, et. al., 2010). However, co-occurring stimuli are not always of the same valence. Sometimes the reason for the co-occurrence is that the object and the affective stimulus are opposite in valence. For instance, the beloved celebrity may co-occur with a new brand on TV because she protests against its manufacturing.

Previous studies (Förderer \& Unkelbach, 2012; Gawronski, Walther, \& Blank, 2005; Hu, Gawronski, \& Balas, 2017; Moran \& Bar-Anan, 2013; Moran, Bar-Anan \& Nosek, 2016) found that when relational qualifiers indicate that the co-occurring stimuli are of opposite valence, the CS acquires the valence opposite to the US. This contrastive effect does not preclude the possibility that, at the same time, the mere CS-US cooccurrence has an independent assimilative effect on evaluation. Perhaps the overall contrastive effect (the CS is evaluated with valence opposite of US valence) is the sum of two opposite effects: an assimilative effect of the CS-US co-occurrence, and a stronger contrastive effect of the inference from the relational information. In the present research, we searched for positive evidence for the persistence of the assimilative effect of cooccurrence when the CS-US relation indicates that they are of opposite valence. 


\section{Theoretical Background}

One of the most influential evaluation models in recent years is the Associative Propositional Evaluation (APE) model (Gawronski \& Bodenhausen, 2006, 2011, 2014, 2018). The APE model distinguishes between associative and propositional processes. Upon learning, associative processes translate the co-activation of mental representations of co-occurring stimuli to a mental association between these representations. Upon expression, associative processes are the activation of a mental representation as a direct result of the activation of an associated mental representation. Propositional learning processes translate external information to mental representations that code specific relations between stimuli and not mere associations. Coding relations between stimuli is essential for making inferences. For instance, the information that co-occurring stimuli are opposite in valence can lead to the inference that an object that co-occurs with negative stimuli is positive. That inference is impossible with associative processes because they do not code relations.

From the APE model's hypotheses follows that exposure to CS-US co-occurrence accompanied by the information that the co-occurring stimuli are of opposite valence would lead to different results for associative processes and propositional processes. Associative processes would form a mental association between the mental representations of the CS and the US. Later, associative processes would lead to the activation of the US when encountering the CS. According to the APE model, that activation would come in the form of a gut reaction, which is the automatic evaluation of the CS. Thus, the automatic evaluation of the CS would be characterized by the US valence. In contrast, propositional processes would allow the inference that the CS is 
characterized by valence opposite of the US valence. According to the APE model, although some propositional processes can occur automatically, inference requires intentional processes and relatively high cognitive capacity (Gawronski \& Bodenhausen, 2018, p. 19). As a result, automatic and deliberate evaluations would be discrepant: the automatic evaluation would reflect an assimilative effect of the co-occurring US valence on the evaluation of the CS, whereas deliberate evaluation would reflect a contrastive effect. If the US is positive, the automatic evaluation of the CS would be positive, and the deliberate evaluation would be negative.

The discrepancy prediction received some empirical support in two studies that exposed participants to CS-US co-occurrence accompanied by relational information (Moran \& Bar-Anan, 2013). Participants observed four families of alien creatures: one started pleasant sounds, one started unpleasant sounds, one ended pleasant sounds, and one ended unpleasant sounds. The automatic evaluation of the creatures reflected the associated stimuli: a preference for the creatures that ended pleasant sounds over the creatures that ended unpleasant sounds. The deliberate evaluation reflected the inference from the relational information: a preference for the creatures that ended unpleasant sounds over the creatures that ended pleasant sounds. These results provide evidence supporting the hypothesis that co-occurring US has an assimilative effect on evaluation even when the CS-US relation indicates that they are of opposite valence

Another finding in Moran and Bar-Anan's (2013) can be interpreted as supporting the hypothesis that co-occurring US has an assimilative effect on evaluation when the CS-US relation indicates that they are of opposite valence. In the self-report evaluation measure, creatures that started pleasant sounds were evaluated more favorably than 
creatures that started unpleasant sounds $\left(\eta_{p}{ }^{2} \mathrm{~s}=0.72,0.66, p \mathrm{~s}<.001, .001\right.$ in Experiments 1 and 2, respectively), and that preference was much larger than the preference for the creatures that ended unpleasant sounds over the creatures that ended pleasant sounds $\left(\eta_{p}{ }^{2} \mathrm{~S}\right.$ $=0.16,0.28, p \mathrm{~s}=.02, .001)$. In other words, overall, beyond the CS-US relation condition, the participants still liked targets that co-occurred with positive valence more than targets that co-occurred with negative valence. This result was not unique: All of the studies that tested the effect of opposition relations between co-occurring stimuli on deliberate evaluation (e.g., Förderer \& Unkelbach, 2012; Gawronski et al., 2005; Hu et al., 2017; Moran et al., 2016) found a main effect of co-occurrence, over and above the effect of the specific relation: people reported more liking of targets that co-occurred with positive stimuli than of targets that co-occurred with negative stimuli.

One possible reason for the effect of co-occurring valence on deliberate evaluation, independent of the effect of inference from relational qualifiers, is that in addition to the effect of inference from the relational information there was also an assimilative effect of co-occurrence on evaluation (Bar-Anan \& Moran, 2018; Moran et al., 2016). The relational information for targets ending positive stimuli pushed the evaluation to the negative side whereas the co-occurrence pushed the evaluation to the positive side. The result of those conflicting forces was an attenuated disliking of the target that ended positive stimuli and an attenuated liking of the target that ended negative stimuli.

There is, however, an alternative to the above interpretation of the attenuated effects of opposition relations on deliberate evaluation, in comparison to the effects of similarity relations. Rather than reflecting an opposite assimilative and contrastive 
effects, the cause of the attenuation might be that some relations are more potent than others in changing evaluation. For example, perhaps relations that diminish the effect of affective stimuli (e.g., end, prevent) are considered weaker evidence for evaluation than relations that facilitate the effect of the affective stimuli (e.g., start, cause). In other words, there is no assimilative effect of the mere link between the CS and the US. Only a weak effect of the inference from the CS-US relation.

To overcome the alternative account, in two experiments, Moran, Bar-Anan, and Nosek (2016) manipulated participants' focus on the CS-US co-occurrence at acquisition. Specifically, intermixed in between the acquisition trials, participants had to indicate with which US each CS co-occurred. The rationale was that a focus on the co-occurrence would increase the strength of the mental CS-US association, thus increasing the assimilative effect. Indeed, the assimilative effect of co-occurrence was stronger when participants had to attend to the co-occurrence during acquisition than when they did not focus on co-occurrence. Difference in potency between similarity and opposition relations cannot explain that finding. Yet, for that finding, experimenter demand might be an alternative account: perhaps the manipulation to focus on the co-occurrence signaled to participants that the researchers believe that the co-occurrence is valid evidence about the CS evaluation, and that the participants should take the co-occurrence into account when they evaluate the CS.

There is another weakness in the evidence supporting the hypothesis that cooccurring US has an assimilative effect on automatic evaluation of the CS which then counteracts and attenuates the effect of inference from an opposition CS-US relation on the deliberate evaluation. The evidence that co-occurring US has an assimilative effect on 
the automatic evaluation of the CS comes from two measures of automatic evaluation: the Implicit Association Test (IAT; Greenwald, McGhee, \& Schwartz, 1998), and the Sorting Paired Features task (SPF; Bar-Anan, Nosek, \& Vianello, 2009). These measures might be sensitive to influences that are independent of evaluative mental representations, like salience asymmetry (e.g., Rothermund \& Wentura, 2004) and recoding processes (e.g., Meissner, \& Rothermund, 2013). Indeed, research published in the current issue (Bading, Stahl, \& Rothermund, 2019) suggests that the IAT results in Moran and BarAnan's (2013) study might reflect factors that have nothing to do with automatic evaluation. Further, a study that tested the effect of the relations cause and prevent between co-occurring stimuli on another indirect measure, the Evaluative Priming Task (EPT; Fazio, Jackson, Dunton, \& Williams, 1995), found a preference according to the relational information and not according to the co-occurrence (Hu et al., 2017; see also Peters \& Gawronski, 2011 for relevant results).

\section{The Present Research}

To summarize the evidence reviewed so far, people's deliberate preference for a CS that co-occurred with positive US over a CS that co-occurred with negative US, an assimilative effect of the US valence on the CS evaluation, disappears and often reverses to a contrast effect when people are informed that the co-occurring stimuli are related in a relation that indicates that they are opposite in their valence. However, the contrast effect in that case is smaller than the assimilative effect found when there is no opposition CSUS relation. The contrast effect might be smaller because the effect of the inference from the CS-US relation is attenuated by an assimilative effect of the mere CS-US link. The best evidence for such an assimilative effect comes from measures of automatic 
evaluation, and from deliberate evaluation by participants who were explicitly instructed to focus on the CS-US co-occurrence at acquisition. However, the former evidence might have reflected factors other than automatic evaluation that influence the tasks used for measuring automatic evaluation. The latter evidence is vulnerable to an experimenter demand account.

The goal of the present research was to search for further support for the hypothesis that CS-US co-occurrence has an assimilative effect on the CS evaluation even when relational information suggests that the co-occurring stimuli are of opposite valence. For that purpose, we examined people's evaluation of the CS under time pressure. Time pressure is a condition that, according to the APE model (Gawronski \& Bodenhausen, 2018), restricts the effect of inference from relational information more than the effect of co-occurrence (activation of US valence). Because time-pressure is not supposed to moderate the automatic assimilative effect of the CS-US co-occurrence on evaluation, time-pressure would increase the relative strength of that effect, in comparison to the effect of inference from the relational information. The MODE model (Motivation and Opportunity as Determinants of Evaluation; Fazio, 2007) offers a similar prediction regarding the effect of time pressure. The MODE model assumes an association, in the person's memory, between the object and evaluation. The associated evaluation reflects a summary of all the associations of the target with valence (including the valence of the co-occurring US). Evaluative response uses the evaluation that is associated with the target as a starting point that can be changed if the person has the motivation and opportunity to change it (Fazio, 2007, p. 623). Time pressure should reduce the opportunity to use the inference from the relational information for correcting 
the effect of co-occurrence on evaluation. Importantly, speeded evaluation is less susceptible than indirect measures of automatic evaluation to factors such as salience asymmetry (e.g., Rothermund \& Wentura, 2004) and recoding (e.g., Meissner, \& Rothermund, 2013).

In the present research, we exposed participants to relational information that indicates that the co-occurring CS and US are of opposite valence (as in Moran and BarAnan, 2013). We presented target characters that started or ended pleasant or unpleasant sounds. We then examined whether the evaluation of the CS that ended the sounds would be closer to the US valence under time pressure than with no time pressure. The strongest evidence for an assimilative effect would be that without time pressure, people would show an evaluation that is in line with the inference from the relational information (more liking of targets that end unpleasant sounds than of targets that end pleasant sounds), but would show evaluation that is in line with the co-occurring valence (the opposite preference) under time pressure. If under time pressure, people would show no preference between the targets that ended the sounds that would be inconclusive evidence. No preference might reflect a decrease in the contrast effect caused by the inference from the CS-US relation down to the same level of the assimilative effect of the CS-US cooccurrence. However, no preference might also reflect an elimination of the effect of the inference, because evaluating under time-pressure is difficult (and no assimilative effect of the CS-US co-occurrence, with or without time-pressure). We conducted three experiments with the same procedure and design. Experiment 1 found inconclusive results which led to modifications in the materials used in Experiment 2. Experiment 2 found strong evidence for the assimilative effect (a preference according to the co- 
occurring valence, under time pressure). To resolve the inconsistency between the results of Experiments 1 and 2, we conducted the final experiment, with a larger sample. ${ }^{1}$

\section{Experiment 1}

\section{Method}

Participants. We planned to run 40 participants in each condition, but accidentally had 37 in the deliberate evaluation condition and 41 in the speeded evaluation condition (total $\mathrm{N}=78,61 \%$ Women, $M_{\text {age }}=24.38, S D_{\text {age }}=2.56$ ). Table 1 presents, for each of the key statistical tests, the effect sizes that the sample of each experiment could detect with power of $80 \%$ and $99 \%$, and the power achieved for the smallest corresponding effect-size found by Moran and Bar-Anan (2013).

Materials. Stimuli were adapted from Moran and Bar-Anan (2013). CSs were 16 drawings of creatures divided equally into four families with distinct visual features and colors (green, red, yellow and purple). The USs were a musical melody and an unpleasant human scream. For the practice evaluation, we used eight drawings of Pokémon creatures (Olson \& Fazio, 2001).

\footnotetext{
${ }^{1}$ We report all data exclusions, manipulations, and measures, and how we determined sample size. Decisions to stop collecting data did not depend on the obtained results. Materials and data are in osf.io/37myb/.
} 
Table 1. Power analysis for the key statistical tests in each experiment

\begin{tabular}{|c|c|c|c|c|c|c|}
\hline \multirow{3}{*}{$\begin{array}{l}\text { Effect of interest } \\
\text { Moran and Bar- } \\
\text { Anan (2013) } \\
\end{array}$} & \multicolumn{3}{|c|}{$\begin{array}{l}\text { Moderation by evaluation-type of the } \\
\text { preference between the families that } \\
\text { ended sounds (partial eta squared) }\end{array}$} & \multicolumn{3}{|c|}{$\begin{array}{l}\text { Preference, among participants who } \\
\text { evaluated under time-pressure*, for } \\
\text { the family that ended positive over the } \\
\text { family the ended negative (Cohen's d) }\end{array}$} \\
\hline & \multicolumn{3}{|c|}{ Experiment 1:.58; Experiment 2:.44 } & \multicolumn{3}{|c|}{ Experiment 1: 0.42; Experiment 2: 0.32} \\
\hline & $\begin{array}{l}80 \% \text { for } \\
\text { detecting } \\
\eta_{\mathrm{p}}^{2} \text { of }\end{array}$ & $\begin{array}{l}99 \% \text { for } \\
\text { detecting } \\
\eta_{\mathrm{p}}^{2} \text { of }\end{array}$ & $\begin{array}{l}\text { Power } \\
\text { achieved } \\
\text { for } \eta_{\mathrm{p}}^{2}=.44\end{array}$ & $\begin{array}{l}80 \% \text { for } \\
\text { detecting d } \\
\text { of }\end{array}$ & $\begin{array}{l}99 \% \text { for } \\
\text { detecting d } \\
\text { of }\end{array}$ & $\begin{array}{l}\text { Power } \\
\text { achieved for } \\
\mathrm{d}=0.32\end{array}$ \\
\hline Experiment 1 & .095 & .195 & $99 \%$ & 0.39 & 0.63 & $64 \%$ \\
\hline Experiment 2 & .095 & .195 & $99 \%$ & 0.39 & .63 & $4 \%$ \\
\hline Experiment 3 & .017 & .035 & $99 \%$ & 0.16 & 0.25 & $99 \%$ \\
\hline $\begin{array}{l}\text { Experiment } 3 \\
\text { (only accurate } \\
\text { participants) }\end{array}$ & .032 & .072 & $99 \%$ & 0.22 & 0.35 & $97 \%$ \\
\hline \multicolumn{7}{|c|}{$\begin{array}{l}\text { Note. Columns } 1,2,4 \text {, and } 5 \text { in the bottom four rows present the effect sizes that the } \\
\text { sample of each experiment could detect with power of } 80 \% \text { and } 99 \% \text {. Columns } 3 \text { and } 6 \text { in } \\
\text { the bottom four rows present the power achieved for the smallest corresponding effect- } \\
\text { size found by Moran and Bar-Anan (2013). *In Moran and Bar-Anan (2013), there was } \\
\text { no evaluation under time-pressure. Instead, we show the effect-size of the assimilative } \\
\text { effect of CS-US co-occurrence on the indirect measures: the IAT (Experiment } 1 \text { ) and the } \\
\text { SPF (Experiment 2). }\end{array}$} \\
\hline
\end{tabular}

Procedure. Participants completed the study in separate cubicles.

Learning. The learning included 10 positive sound $\left(\mathrm{US}_{\mathrm{pos}}\right)$ trials and 10 negative sound $\left(\mathrm{US}_{\mathrm{neg}}\right)$ trials separated by a soft ticking sound that played for 10-15 seconds. Each trial began with a presentation of the "starting" creature (CS) appearing in silence for $500 \mathrm{~ms}$. Next, the auditory stimulus played for a randomly selected duration of 10-30 seconds. The "starting" creature remained on the screen for the first two seconds of the playback. Then the screen remained blank until the "ending" creature (CS) appeared for the last two seconds of the auditory playback and remained on the screen for another $500 \mathrm{~ms}$ of silence. When the screen turned blank, the ticking sound commenced until the next trial. We instructed participants to learn 
which family performed each of the four actions for a later memory test. We counterbalanced between participants which family performed each of the four roles.

Evaluation. Next, participants reported their evaluations of the 16 creatures. For each of the 16 questions (randomly ordered), the creature appeared above the instructions to "rate how positive or negative the creature is" by clicking the mouse on an analog visual scale that ranged from negative (coded 0) to positive (coded 300). In the deliberation condition, we instructed participants to think carefully about their judgment and the scale appeared only 10 seconds after the creature appeared. In the speeded condition, we instructed participants to respond as quickly as possible, and they had $1,500 \mathrm{~ms}$ to respond after the creature appeared. After $1,500 \mathrm{~ms}$ with no response, the creature disappeared and a "please response faster" message appeared for 500ms.

Before evaluating the creatures, participants practiced by evaluating eight unrelated creatures. To provide similar delay between learning and evaluation, all participants practiced both rating types. In the deliberation condition, participants first rated the eight creatures under time pressure, and then four of the creatures under the deliberation rules. The practice order was the opposite in the speeded condition.

Memory. In the four final questions, participants indicated what action each family performed (one family at a time).

Design. Excluding counterbalance of stimuli assignment, the design was 2 (US valence: positive, negative; within participants) $\times 2$ (Relational information: start, end; within participants) $\times 2$ (Evaluation time: speeded, deliberate; between participants). 


\section{Results}

The mean ratings are illustrated in Figure 1. We mainly focused on the evaluation of the targets that ended the sounds, because for these targets the effect of co-occurrence and the effect of the inference from the relational information, are opposite in valence. We submitted the ratings of the targets that ended the sounds to a 2 (US valence) $\times 2$ (evaluation) ANOVA. The ANOVA found an effect for US valence, $F(1,76)=4.07, p=$ $.047, \eta_{p}^{2}=.05$, reflecting a more positive evaluation of CSs that co-occurred with unpleasant noise $(M=165.91, S D=67.49)$ than of CSs that co-occurred with pleasant music $(M=145.60, S D=55.36)$. Of main interest was whether the effect of US-valence would be moderated by the evaluation time condition. That moderation was not significant, $F(1,76)=1.93, p=.169, \eta_{p}{ }^{2}=.02$. Nevertheless, the pattern of the results was compatible with an increase in the strength of the assimilative effect of the US valence relative to the contrastive effect, under time pressure in comparison to the evaluation with no time pressure. When participants evaluated the families that ended the US, they evaluated more favorably CSs that co-occurred with $\mathrm{US}_{\text {neg }}$ (family members that ended the unpleasant noise) than CSs that co-occurred with $\mathrm{US}_{\mathrm{pos}}$ (family members that ended the pleasant music) only if participant had time for deliberate evaluation, $F(1,36)$ $=5.27, p=.027, \eta_{p}{ }^{2}=.12, B F_{10}=5.15$, but not when participants had only $1.5 \mathrm{~s}$ to evaluate each creature, $F(1,40)=0.22, p=.643, \eta_{p}{ }^{2}<.001, B F_{10}=0.24$. As we report in the supplement ( $\underline{\text { osf.io/k3z65/) }}$, in all three experiments, even under time-pressure, participants always reported preference for the family that started the music over the family that started the noise $\left(\eta_{p}^{2}=.66-.73\right.$, in the three experiments). That suggests that the time-pressure did not eliminate people's ability to judge stimuli. 
Memory. Most participants $(n=74)$ remembered the roles of all families.

Participants in the deliberate evaluation condition did not have better memory $(M=0.92$, $S D=0.28)$ than participants in the speeded condition $(M=0.98, S D=0.16)$, ruling out the possibility that difference in memory accuracy could explain the results.

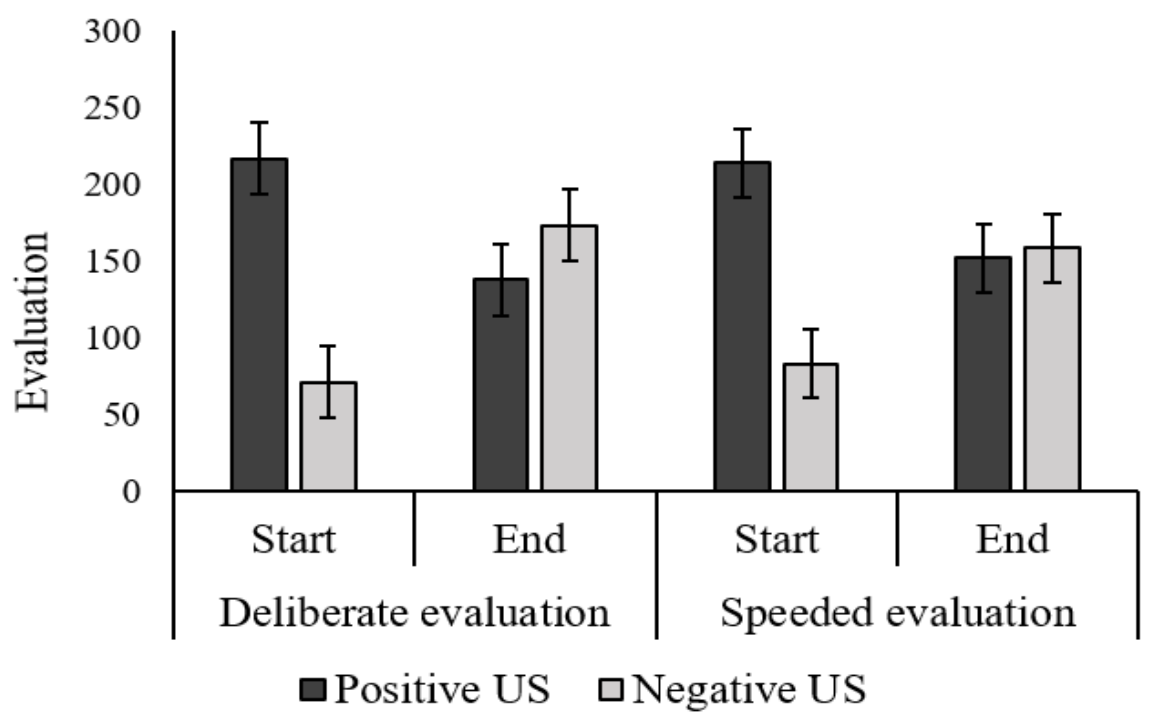

Figure 1. Experiment 1: mean evaluation scores as a function of US valence (positive vs. negative), relational information (start vs. end) and evaluation time (speeded vs. deliberate). Error-bars are 95\% confidence intervals (calculated based on Jarmasz \& Hollands, 2009).

\section{Discussion}

Experiment 1 found that the CS evaluation was closer to the US valence under time pressure than with no time pressure. However, evaluation under time pressure 
showed no preference between the families that ended the sounds. In the present context, this is an inconclusive finding. The finding is compatible with the hypothesis that the contrastive effect of inference from a CS-US opposition relation weakened under time pressure, relative to an assimilative effect of CS-US co-occurrence. However, it is also compatible with the possibility that CS-US co-occurrence has no effect on the evaluation; Time pressure simply eliminated the effect of inference.

When we explored the effect of specific stimuli in Experiment 1, we found that two of the families (the green and the red families) were evaluated under time pressure as unfavorable (see osf.io/m5rgh/ for full details). We speculated that time pressure might elicit negative evaluation of these two particular colors, regardless of the information previously provided of the families. Therefore, we conducted Experiment 2 as a direct replication of Experiment 1, only replacing these two potentially problematic stimuli.

\section{Experiment 2}

\section{Method}

Participants. We planned to run 40 participants in each condition, inadvertently ran four more, but then lost the data of five participants due to a program error. The final sample included 79 participants (77\% Women, $M_{\text {age }}=23.06, S D_{\text {age }}=1.54$ ).

Materials and procedure. The materials and procedure were identical to Experiment 1 except that we replaced the green and red families with new families of creatures (orange and brown). 


\section{Results}

The mean ratings are illustrated in Figure 2. We submitted the ratings of the targets that ended the sounds to a 2 (US valence) $\times 2$ (Evaluation time) ANOVA. That ANOVA found an effect for evaluation time, $F(1,77)=6.51, p=.013, \eta_{p}{ }^{2}=.08$, reflecting a more positive evaluation when participant had time for deliberate evaluation $(M=165.28, S D=36.60)$ than when they had only 1.5 s for evaluation $(M=143.02, S D=$ 40.63). Importantly, there was an interaction between US-valence and evaluation time condition, $F(1,77)=10.07, p=.002, \eta_{p}{ }^{2}=.12$. This interaction reflected the hypothesized discrepancy: a more positive evaluation of CSs that co-occurred with $\mathrm{US}_{\text {neg }}$ (the family that ended the unpleasant noise) than of CSs that co-occurred with $\mathrm{US}_{\text {pos }}$ (the family that ended the pleasant music) when participant had time for deliberate evaluation, $F(1,37)=4.58, p=.039, \eta_{p}{ }^{2}=.11, B F_{10}=4.57$, but the opposite preference-a more positive evaluation of CSs that co-occurred with $\mathrm{US}_{\mathrm{pos}}$ (the family that ended the pleasant music) than of CSs that co-occurred with $\mathrm{US}_{\text {neg }}$ (the family that ended the unpleasant noise) - when participants had only $1.5 \mathrm{~s}$ to evaluate each creature, $F(1,40)=5.59, p=$ $.023, \eta_{p}^{2}=.12, B F_{10}=5.42$.

Memory. Most participants $(n=73)$ remembered the roles of all families. Participants in the deliberate evaluation condition did not have better memory $(M=0.92$, $S D=0.27)$ than participants in the speeded condition $(M=0.93, S D=0.26)$, ruling out the possibility that difference in memory accuracy could explain the results. 


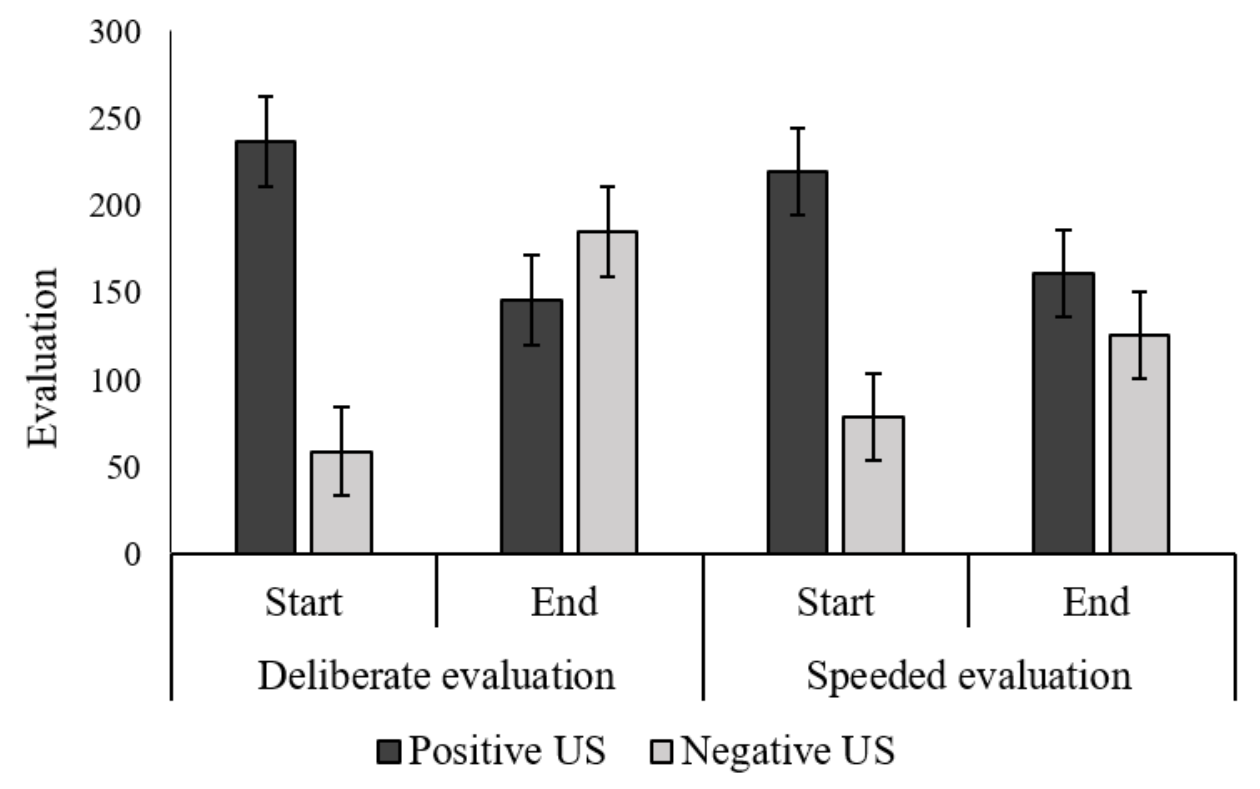

Figure 2. Experiment 2: mean evaluation scores as a function of US valence (positive vs. negative), relational information (start vs. end) and evaluation time (speeded vs. deliberate). Error-bars are 95\% confidence intervals (calculated based on Jarmasz \& Hollands, 2009).

\section{Discussion}

The results of Experiment 2 provide strong support for the hypothesis that the valence of a co-occurring US has an assimilative effect on the evaluation of the CS even when participant know that the co-occurring CS and US are of opposite valence. When participants had time for deliberate evaluation, their evaluation was in line with the inference from the relational information (more favorable evaluation of targets that end unpleasant sounds than of targets that end pleasant sounds). By contrast, when participants had only $1.5 \mathrm{~s}$ for evaluation, their evaluation was in line with the cooccurring valence (the opposite preference). Because that clear evidence for an 
assimilative effect on speeded evaluation was not found in Experiment 1, we conducted Experiment 3, repeating the same procedure with a larger sample to increase the accuracy of the estimation of the effects in that procedure.

\section{Experiment 3}

\section{Method}

Participants. We pre-registered the target sample size, exclusion rules, and analysis plans (https://osf.io/ce4y2/). As planned, 470 participants participated online via the Prolific Academic website (https://prolific.ac). Data from four participants was not recorded. We excluded six participates who did not pay attention to the induction task. The final sample included 460 participants (55\% Women, $M_{\text {age }}=36.68, S D_{\text {age }}=11.65$ ).

Materials and procedure. The materials and procedure were similar to Experiment 2, except for the following modifications, required for an Internet study. At acquisition, the positive and negative sounds were played for a randomly selected duration of 6-18 seconds. To ensure that participants listened to the sounds, we added at acquisition catch-trials with a secondary task. In those trials, no creatures were shown. Instead, the sound was a word, followed by a display of three words, from which the participants had to choose the word they just heard. Participants first completed three catch-trials, and then 10 positive sound, 10 negative sound and 10 catch-trials, ordered randomly. In the speeded evaluation task, participants had 2 s to respond after the creature appeared, instead of $1,500 \mathrm{~ms} .{ }^{2}$ The evaluation scale ranged from "extremely negative"

\footnotetext{
${ }^{2}$ A shorter duration in a pilot study resulted with low success-rate of rating the creatures on time.
} 
(0) to "extremely positive" (100). For the practice evaluation, we used eight drawings of novel creatures similar to the CSs.

\section{Results}

We conducted two analyses. One including all the participants and one including only participants who responded correctly to all four memory questions about the roles of the families. Because we were concerned that some paid participants over the Internet might not be motivated to pay attention to the tasks, we preregistered our plan to trust the results of the accurate sample ( $52 \%$ of the participants, $\mathrm{n}=240,54 \%$ Women, $M_{\text {age }}=$ $\left.35.74, S D_{\text {age }}=10.85\right)$ more than the results of the whole sample, if the results would differ. The result of all the participants are reported in the online supplement (osf.io/bw698/).

Accurate participants. The mean ratings are illustrated in Figure 3. We submitted the ratings of the CSs that ended the sounds to a 2 (US valence) $\times 2$ (Evaluation time) ANOVA. That ANOVA found a main effect of evaluation time, $F(1$, 238) $=5.88, p=.016, \eta_{p}{ }^{2}=.02$, reflecting overall more positive ratings when participants had time for deliberate evaluation $(M=56.81, S D=11.25)$, than when they had only $2 \mathrm{~s}$ $(M=53.41, S D=10.44)$. The effect of US valence was also significant, $F(1,238)=6.41$, $p=.012, \eta_{p}{ }^{2}=.03$, reflecting more positive evaluation of CSs that ended the unpleasant noise $(M=56.78, S D=17.38)$ than of CSs that ended the pleasant music $(M=53.29, S D$ = 14.14). Importantly, the effect of US valence was further moderated by the evaluation time condition, $F(1,238)=9.98, p=.002, \eta_{p}^{2}=.04$. When participants had time for deliberate evaluation, they rated CSs that ended the unpleasant noise more favorably than CSs that ended the pleasant music, $F(1,114)=14.01, p<.001, \eta_{p}{ }^{2}=.11, B F_{10}=302.66$. 
By contrast, when participants had only $2 \mathrm{~s}$ to evaluate each creature, there was no significant preference between the two CSs, $F(1,124)=0.23, p=.633, \eta_{p}{ }^{2}<.01, B F_{10}=$ 0.15. These results are similar to the results of Experiment 1.

As reported in the online supplement, the results of the whole sample were similar with one crucial difference: in that sample, even when there was no time pressure, participants did not evaluate the creatures that ended the unpleasant noise more favorably (or more unfavorably) than the creatures that ended the pleasant sound. These results suggest that the whole sample was contaminated by unmotivated participants who failed to learn the roles of the families well enough ${ }^{3}$. As pre-registered, we base our interpretation of the result on the sample of participants who remembered accurately the roles of all four families.

\section{Discussion}

Experiment 3 found that the evaluation of the CS was closer to the US valence under time pressure than with no time pressure. However, under time pressure there was no preference between the families that ended the sounds. Thus, like in Experiment 1, Experiment 3's results were inconclusive.

\footnotetext{
${ }^{3}$ In Experiment 1 and 2, analyzing only accurate participants found the same results as the analyses that included all the participants (see osf.io/n7wy6/ for full details).
} 


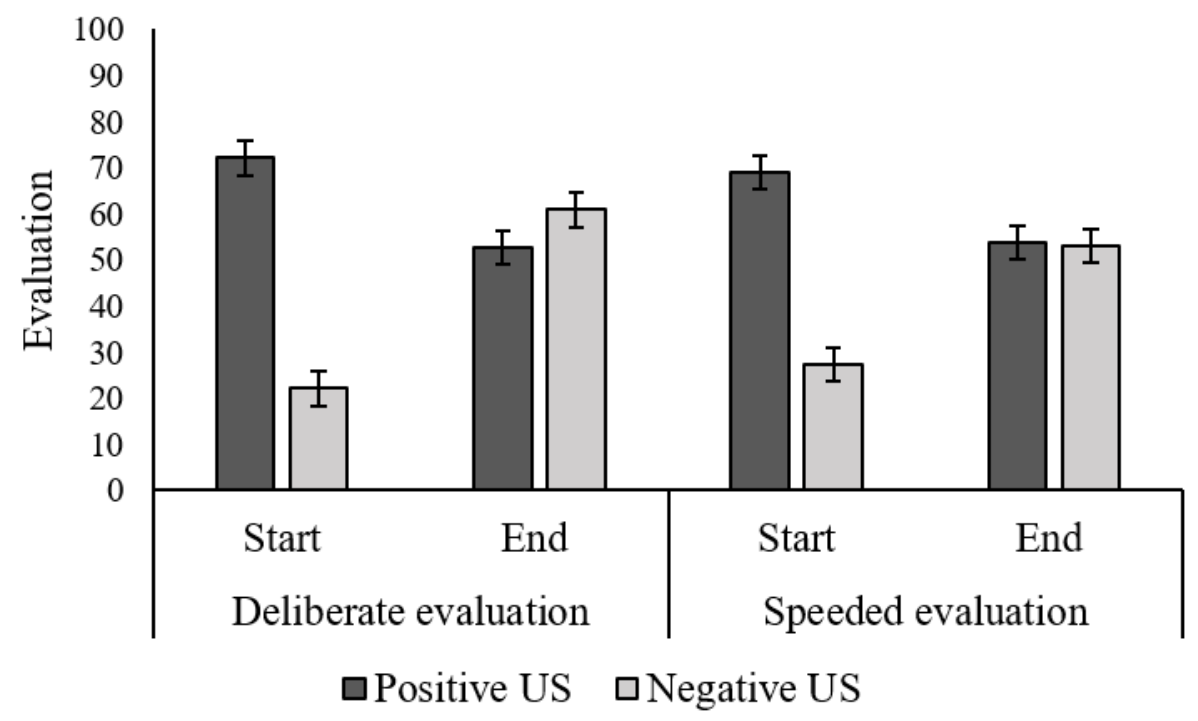

Figure 3. Accurate participants in Experiment 3: mean evaluation scores as a function of US valence (positive vs. negative), relational information (start vs. end) and evaluation time (speeded vs. deliberate). Error-bars are 95\% confidence intervals

\section{General Discussion}

The goal of the present research was to test the hypothesis that after a CS-US cooccurrence, the US valence has an assimilative effect on the CS evaluation even if relational information suggests that the co-occurring stimuli are of opposite valence (BarAnan \& Moran, 2018). For that purpose, we examined the effect of exposure to CS-US co-occurrence, accompanied by the information that the CS ends the US, on the evaluation of the CS under time-pressure. According to the APE model (Gawronski \& Bodenhausen, 2018), time-pressure, a condition that does not allow recruiting much cognitive resources, restricts the effect of inference from relational information but does not restrict the automatic assimilative effect of the CS-US co-occurrence on evaluation. If 
under time pressure, participants show preference that is in line with the valence of the US that co-occurred with each CS, that would be strong support for the hypothesis.

We found strong support for the assimilative effect of the US valence on the speeded CS evaluation only in Experiment 2. In all three experiments, when participants evaluated the targets with no time pressure, they rated targets that co-occurred with unpleasant sounds because they ended those sounds more favorably than targets that ended (and co-occurred with) pleasant sounds. That is a contrast effect of the US valence on the CS evaluation, probably as a result of inference from the CS-US relation (CS ends $U S)$. Under time pressure, that contrast effect disappeared in all three experiments, and was significantly different (in Experiments 2 and 3) from the preference shown by participants who evaluated the targets without time pressure. However, only in Experiment 2, participants' evaluation of the targets that ended positive sounds was significantly more favorable than their evaluation of targets that ended negative sounds. That effect strongly supports the hypothesis that the mere CS-US co-occurrence had an effect on CS evaluation, and improves the likelihood of the hypothesis that this assimilative effect occurs even in deliberate evaluation without time pressure, counteracting the effect of the inference from the CS-US relation, and leading to an overall attenuated contrast effect of the US valence on the CS evaluation.

The strong evidence in Experiment 2 is seriously challenged, however, by the results of Experiments 1 and 3. In those experiments, under time-pressure, there was no preference for any CS over the other. Under time pressure, the contrast effect was attenuated and nullified. That nullification could reflect a decrease in the contrast effect caused by the inference from the CS-US relation down to the same level of the 
assimilative effect of the CS-US co-occurrence, which, supposedly, was not affected by the time-pressure. Yet, it can also reflect complete elimination of the effect of the inference, without any effect of the CS-US co-occurrence on the CS evaluation, regardless of time-pressure or its absence. The only evidence against that alternative is that even under time-pressure, in all three experiments, the participants evaluated the creatures that started pleasant music much more favorably than the creatures that started the unpleasant noise $\left(\eta_{\mathrm{p}}{ }^{2}=.66-.73\right)$. In fact, that difference was moderated by timepressure only in Experiment 3, but not in Experiments 1 and 2. This finding decreases the likelihood of the possibility that time-pressure eliminated completely people ability to report their evaluation between creatures. Nevertheless, because it might be more difficult to evaluate the creatures that ended the sounds, perhaps time-pressure had a stronger effect on that evaluation. Therefore, the alternative account is still plausible. Overall, in a mini meta-analysis ${ }^{4}$ of the effects found in the three experiments, for the difference between the families that ended the sounds, under time pressure, we found that this effect was not reliably different than zero, $d=0.13, Z=1.28, p=.198$. Therefore, we conclude that the results of the present research do not provide positive evidence that a co-occurring US has an assimilative effect on the CS evaluation, when people know that the CS-US relation is opposition.

The inconclusiveness of the present results leaves the hypothesis that we tested with only some support from prior research. We think that the best supporting evidence comes from an experiment (Moran, Bar-Anan, \& Nosek, 2016, Experiment 5) that used

\footnotetext{
${ }^{4}$ Computed using the Metafor package in $\mathrm{R}$ (Viechtbauer, 2010). Following the recommendations by Lakens (2013), Cohen's d was calculated using SD pooled.
} 
the same learning procedure we used in the present research, and manipulated participants' focus on the CS-US co-occurrence at acquisition. Specifically, in the cooccurrence focus condition, intermixed in between the acquisition trials, participants had to indicate with which US each CS co-occurred. After the acquisition, all the participants evaluated all the creatures (with no time pressure). In the co-occurrence focus condition (but not in the control conditions), participants evaluated the creatures that ended positive sounds more favorably than creatures that ended negative sounds. Importantly, the participants remembered correctly the roles of the families. In other words, when participants focused on the CS-US co-occurrence during acquisition, the overall effect was an assimilative effect of the US valence on the evaluation of the CS, despite participants' clear knowledge of the CS-US opposition relation. The only weakness in that evidence is that perhaps the questions about the mere existence of a relation between the creatures, with no regard to the type of the relation, changed participants' usual inference process to inference that reached an unusual conclusion rather than strengthened an assimilative effect that always occurs.

In the present research we tried to strengthen the relative effect of the hypothesized assimilative effect of the US on the CS evaluation by restricting the available cognitive resources that might be needed for the processes that underlie the competing effect of inference from the CS-US relation. Conclusive results (a consistent assimilative effect under time pressure) would have been compatible with the assumption that the assimilative effect also occurs and influences evaluation when people have ample cognitive resources. Yet, even conclusive results would have been vulnerable to the possibility that the assimilative effect occurs under time-pressure conditions but not when 
people have the cognitive resources needed to completely reject and cancel (rather than counteract) the assimilative effect. Even under that limitation, testing speeded evaluation seems important because, currently, there is probably not enough evidence for a strong conclusion that co-occurring US has an assimilative effect on automatic CS evaluation when people also know that the reason for the CS-US co-occurrence is an opposition relation. Like in Experiments 1 and 3 of the present research, quite a few studies have found no preference - automatic or deliberate — between the CS and the US when people know that these have an opposition relation (Hughes, Ye, \& De Houwer, 2018; Zanon, De Houwer, \& Gast, 2012; Zanon, De Houwer, Gast, \& Smith, 2014). Only very few studies (Gregg, Seibt, \& Banaji, 2006; Moran \& Bar-Anan, 2013; Wyer, 2016) have found an overall assimilative effect of the US on the automatic evaluation of the CS when CS and US had opposition relations. However, conceptual or direct replications of some of these studies (Gregg, Seibt, \& Banaji, 2006; Wyer, 2016) found different results (Moran, Bar-Anan, \& Nosek, 2017), and alternative interpretations (Bading, Stahl, \& Rothermund, 2019) were suggested for other evidence (Moran \& Bar-Anan, 2013). Therefore, the inconclusiveness of the present results leaves in doubt the hypothesis that CS-US co-occurrence leads to an assimilative effect of the US valence on the CS evaluation (deliberate or automatic), even when people know that the CS-US relation is opposition.

\section{Theoretical Implications and Further Research Directions}

According to the APE model, the evaluation of the CS under low cognitive resources is sensitive to the mere CS-US co-occurrence more than to inferential processes. The decrease, under time-pressure, in the contrast effect of the US valence is 
compatible with that assumption. However, as we already discussed, the present results do not provide strong evidence for the assimilative effect of the CS-US co-occurrence because they are open to an alternative account. Although they do not provide strong support for the APE model, the results of Experiment 1 and 3 are not problematic for the APE model. A simple account for the absence of an overall assimilative effect of the US valence under time pressure is that the time pressure only decreased but did not eliminate people's ability to make inferences. This account seems especially reasonable for Experiment 3, in which we allowed participants a slightly longer time to respond (2 seconds, in comparison to 1.5 seconds in Experiments 1 and 2), to avoid excluding too many participants who were too slow to respond. To overcome this problem, further research could manipulate the response deadline under time pressure within participants (using multiple target stimuli). The purpose of that manipulation would be to find, for each participant, the response deadline that eliminates the effect of inference but still allows for the effect of associative evaluation processes.

Another account for the results of Experiments 1 and 3 that does not challenge the APE model is that before the evaluation, perhaps in acquisition, the participants have already made the inference about the true valence of the CS, and that inference formed mental associations between the CS and the opposite valence of the US (Bar-Anan \& Moran, 2018). In that case, an evaluation under low cognitive resources would reflect two opposing mental associations, an association of the CS with the US valence formed due to the CS-US co-occurrence, and an association of the CS with the opposite valence, formed due to an inference from the CS-US relation. That would explain the difficulty in finding an overall assimilative effect, even under time-pressure. Compatible with that 
possibility, previous studies that used a more direct manipulation of relational information (presented relational qualifiers simultaneously with the CS-US pairing), perhaps allowing stronger inference from the relational information during encoding, found a strong effect of relational information on both deliberate and automatic evaluation (Hu et al., 2016; Peters \& Gawronski, 2006). Further research could employ manipulations to directly influence encoding processes at acquisition and test whether these manipulations influences the automatic evaluation of the CS.

Some might consider the paucity of evidence in support of an effect of the CS-US mere co-occurrence on automatic evaluation favorable for the propositional perspective on evaluation and evaluative learning (De Houwer, 2018; Mitchell, De Houwer, \& Lovibond, 2009). According to the propositional perspective, the mental representations formed in acquisition and activated in expression are always propositional (they represent relations and not mere links between concepts). If there is hardly any evidence for automatic evaluation that does not reflect the expected inference from the CS-US relation, it might seem more likely that encoding, retrieval, and judgment processes all operate with propositional mental representations. However, it should be noted that the propositional perspective is, in fact, immune to evidence of automatic evaluation that does not reflect the expected inference from the CS-US relation because that perspective allows for the effects of only a part of the mental representation on behavior. For example, even if the mental representation that one stores about blue creatures is blue creatures end good, exposure to blue creatures might activate positive valence as a result of a similarity-based retrieval, and lead to automatic positive evaluative response, by processes that do not involve inference (because of that, it is virtually impossible to 
falsify the propositional perspective, De Houwer, 2018). The wide agreement, across theoretical models, that automatic evaluation processes can reflect the mere link between object and valence even when people know that the link is due to an opposition relation, further emphasizes the importance of finding replicable convincing evidence of that effect, a worthy target for further research. The absence of strong evidence for such an effect might inspire a shift in the understanding of the factors that influence automatic evaluation.

All the perspectives mentioned so far can be used to generate an account that would explain why the contrast effect of a US valence that is opposite of a co-occurring CS on the deliberate evaluation of the CS is weaker than the assimilative effect of a US that has a similarity relation with a co-occurring CS. Because both the propositional perspective and the APE model assume that deliberate evaluation is governed by propositional processes, accounts compatible with these models might focus on the possibility that people consider some CS-US relations as weaker evidence for evaluation than others (Hughes et al., in press). As an alternative, Bar-Anan and Moran (2018) suggested the hypothesis that deliberate evaluation of a target object treats the activation of valence linked with the target as valid evidence that the valence characterizes the object, regardless of the reason that linked the valence with the object. In a similar vein, a memory-based approach to EC (Stahl \& Aust, 2018) might suggest that because people remember the US valence and the CS-US specific relation, an evaluative judgment about the CS would retrieve both the information about the US valence and the specific relation between the CS and the US, resulting with a behavioral response that would reflect a combination of these retrieved memories. Importantly, at this time, there is no account for 
that finding that has received strong and conclusive empirical support. Further understanding of that finding is likely to add valuable knowledge about the fundamentals of human judgment. 


\section{References}

Bading, K., Stahl, C., \& Rothermund, K. (2019). Why a standard IAT effect cannot provide evidence for associative learning: The role of similarity construction. Unpublished manuscript [please note that we think that it is likely to appear in the present special issue]

Bar-Anan, Y., \& Moran, T. (2018). Simple First: A Skeleton for an Evaluative Learning Model. Social Psychological Bulletin, 13, e28761.

Bar-Anan, Y., Nosek, B. A., \& Vianello, M. (2009). The sorting paired features task: A measure of association strengths. Experimental Psychology, 56(5), 329-343.

De Houwer, J. (2018). Propositional models of evaluative conditioning. Social Psychological Bulletin, 13, e28046.

De Houwer, J., Thomas, S., \& Baeyens, F. (2001). Association learning of Likes and dislikes: A review of 25 years of research on human evaluative conditioning. Psychological Bulletin, 127, 853-869.

Fazio, R. H. (2007). Attitudes as object-evaluation associations of varying strength. Social cognition, 25(5), 603-637.

Fazio, R. H., Jackson, J. R., Dunton, B. C., \& Williams, C. J. (1995). Variability in automatic activation as an unobtrusive measure of racial attitudes: a bona fide pipeline?. Journal of Personality and Social Psychology, 69(6), 1013-1027.

Förderer, S., \& Unkelbach, C. (2012). Hating the cute kitten or loving the aggressive pitbull: EC effects depend on CS-US relations. Cognition and Emotion, 26(3), 534540. 
Gawronski, B., \& Bodenhausen, G. V. (2006). Associative and prepositional processes in evaluation: An integrative review of implicit and explicit attitude change. Psychological Bulletin, 132(5), 692-731.

Gawronski, B., \& Bodenhausen, G. V. (2011). The associative-propositional evaluation model: Theory, evidence, and open questions. Advances in Experimental Social Psychology, 44, 59-127.

Gawronski, B., \& Bodenhausen, G. V. (2014). The associative- propositional evaluation model: Operating principles and operating conditions of evaluation. In J. W. Sherman, B. Gawronski, \& Y. Trope (Eds.), Dual-process theories of the social mind (pp. 188 -203). New York, NY: Guilford Press.

Gawronski, B., \& Bodenhausen, G. V. (2018). Evaluative conditioning from the perspective of the associative-propositional evaluation model. Social Psychological Bulletin, 13, e28024.

Gawronski, B., Walther, E., \& Blank, H. (2005). Cognitive consistency and the formation of interpersonal attitudes: Cognitive balance affects the encoding of social information. Journal of Experimental Social Psychology, 41(6), 618-626.

Greenwald, A. G., McGhee, D. E., \& Schwartz, J. K. L. (1998). Measuring individual differences in implicit cognition: The Implicit Association Test. Journal of Personality and Social Psychology, 74(6), 1464-1480.

Gregg, A. P., Seibt, B., \& Banaji, M. R. (2006). Easier done than undone: asymmetry in the malleability of implicit preferences. Journal of personality and social psychology, 90(1), 1-20. 
Hofmann, W., De Houwer, J., Perugini, M., Baeyens, F., \& Crombez, G. (2010). Evaluative conditioning in humans: a meta-analysis. Psychological Bulletin, 136(3), 390-421.

Hu, X., Gawronski, B., \& Balas, R. (2017). Propositional versus dual-process accounts of evaluative conditioning: I. The effects of co-occurrence and relational information on implicit and explicit evaluations. Personality and Social Psychology Bulletin, 43(1), 17-32.

Hughes, S., Ye, Y., \& De Houwer, J. (2018). Evaluative conditioning effects are modulated by the nature of contextual pairings. Cognition and Emotion, doi: $10.1080 / 02699931.2018 .1500882$

Hughes, S., Ye, Y., Van Dessel, P., \& De Houwer, J. (in press). When people co-occur with good or bad events: Graded effects of relational qualifiers on Evaluative Conditioning. Personality and Social Psychology Bulletin.

Jarmasz, J., \& Hollands, J. G. (2009). Confidence intervals in repeated-measures designs: The number of observations principle. Canadian Journal of Experimental Psychology, 63(2), 124-138.

Lakens, D. (2013). Calculating and reporting effect sizes to facilitate cumulative science: A practical primer for t-tests and ANOVAs. Frontiers in Psychology, 4. doi:10.3389/fpsyg.2013.00863

Meissner, F., \& Rothermund, K. (2013). Estimating the contributions of associations and recoding in the Implicit Association Test: The ReAL model for the IAT. Journal of Personality and Social Psychology, 104(1), 45-69. 
Mitchell, C. J., De Houwer, J., \& Lovibond, P. F. (2009). The propositional nature of human associative learning. Behavioral and Brain Sciences, 32(2), 183-198.

Moran, T., \& Bar-Anan, Y. (2013). The effect of object-valence relations on automatic evaluation. Cognition and Emotion, 27(4), 743-752.

Moran, T., Bar-Anan, Y., \& Nosek, B. A. (2016). The assimilative effect of cooccurrence on evaluation above and beyond the effect of relational qualifiers. Social Cognition, 34, 343-356.

Moran, T., Bar-Anan, Y., \& Nosek, B. A. (2017). The effect of the validity of cooccurrence on automatic and deliberate evaluations. European Journal of Social Psychology, 47(6), 708-723.

Olson, M. A., \& Fazio, R. H. (2001). Implicit attitude formation through classical conditioning. Psychological Science, 12(5), 413-417.

Peters, K. R., \& Gawronski, B. (2011). Are we puppets on a string? Comparing the impact of contingency and validity on implicit and explicit evaluations. Personality and Social Psychology Bulletin, 37(4), 557-569.

Rothermund, K., \& Wentura, D. (2004). Underlying processes in the implicit association test: dissociating salience from associations. Journal of Experimental Psychology: General, 133(2), 139-165.

Stahl, C., \& Aust, F. (2018). Evaluative conditioning as memory-based judgment. Social Psychological Bulletin, 13, e28589.

Viechtbauer, W. (2010). Conducting Meta-Analyses in R with the metafor Package. Journal of Statistical Software, 36(3), 1-48. 
Wyer, N. A. (2016). Easier done than undone... by some of the people, some of the time: The role of elaboration in explicit and implicit group preferences. Journal of Experimental Social Psychology, 63, 77-85.

Zanon, R., De Houwer, J., \& Gast, A. (2012). Context effects in evaluative conditioning of implicit evaluations. Learning and Motivation, 43(3), 155-165.

Zanon, R., De Houwer, J., Gast, A., \& Smith, C. T. (2014). When does relational information influence evaluative conditioning?. The Quarterly Journal of Experimental Psychology, 67(11), 2105-2122. 